\title{
Valoración del riesgo psicosocial en las enfermeras de nefrología de los hospitales de Sevilla
}

\author{
Eugenio Mesa de la Torre* \\ Antonio Gálvez Díaz* \\ Manuel Ángel Calvo Calvo* \\ $M^{a}$ Dolores Vázquez Franco* * \\ Rafael Castilla Requena* * \\ Antonio Luque Cid**
}

* Servicio de Nefrología del Hospital General

Universitario Virgen del Rocío

**Servicio de Nefrología del Hospital

Universitario Virgen Macarena

Sevilla

\section{RESUMEN}

El objetivo general de este trabajo fue realizar una evaluación psicosocial del personal de enfermería de las unidades de nefrología de los hospitales "Virgen del Rocío" y "Virgen Macarena" de Sevilla, siendo los objetivos específicos del estudio describir la situación de este grupo en cuanto a clima laboral, tipo de conflictos del grupo, gestión de los conflictos, relación con el mando inmediato y bases de poder, acoso laboral y comparación entre las unidades de ambas instituciones. El estudio se realizó evaluando 66 encuestas contestadas por el personal de enfermería de los servicios de nefrología, de ambos hospitales. Las conclusiones obtenidas fueron las siguientes:

1. El clima laboral es una mezcla entre clima de apoyo y reglas.

2. El tipo de conflicto que predomina en la organización es de tareas sobre el afectivo.

3. El estilo de gestión para solucionar los conflictos es el de integración.

4. Las bases de poder están divididas entre formales $e$ informales y el mando no es autoritario.

5. El acoso laboral es casi inexistente, siendo éste sobre la función laboral del trabajador, lo que provoca muy baja incidencia somática en el individuo.

Correspondencia:

Eugenio Mesa de la Torre

C/ Emilio Lemos, $n^{\circ}$ 1-2--B

41020 Sevilla
6. Las diferencias halladas entre las dos unidades no son significativas.

PALABRAS CLAVE: RIESGO PSICOSOCIAL

ENFERMERÍA

NEFROLOGÍA

\section{EVALUATION OF THE PSYCHOSOCIAL RISK IN NEPHROLOGY NURSES IN HOSPITALS IN SEVILLE}

\section{SUMMARY}

The general aim of this work was to conduct a psychosocial assessment of nursing staff in the nephrology units of the hospitals Virgen del Rocío and Virgen Macarena in Seville, the specific aims of the study being to describe the situation of this group as regards the working environment, type of group conflicts, conflict management, relations with immediate superiors and power bases, harassment and comparison between the units in the two hospitals. The study was carried out by assessing 66 surveys to which nephrology service nursing staff in both hospitals had responded. The conclusions obtained were as follows:

1. The working environment is a mixture of support and rules.

2. The predominating type of conflict in the organization relates to tasks rather than emotional. 
3. The management style to resolve conflicts is integration.

4. The power bases are divided between formal and informal and command is not authoritarian.

5. Harassment is almost non-existent, and is directed at the employee's work, which causes a very low somatic incidence on the individual.

6. The differences found between the two units are not significant.

KEY WORDS: PSYCHOSOCIAL RISK

NURSING

NEPHROLOGY

\section{INTRODUCCIÓN}

Las alteraciones psicológicas y dentro de ellas el estrés, constituyen una de las principales causas de incapacidad laboral. Según nuestra experiencia la Enfermería se considera habitualmente una ocupación muy estresante con prevalencias altas de trastornos psicológicos relacionados con el estrés.

Este problema tan extendido supone un gran coste tanto para el individuo, al provocar incapacidad física y mental, como para las empresas y la sociedad, debido al absentismo, al bajo rendimiento y a los gastos sanitarios. Según Neffa, las condiciones y medio ambiente de trabajo están constituidas por un conjunto de variables que, en el nivel de la sociedad en su conjunto, de manera directa o indirecta van a influir sobre la vida y la salud de los trabajadores; influencia que va a depender en cada caso de las respectivas capacidades de adaptación y de resistencia a los factores de riesgo( ${ }^{(1)}$. Esas variables, entre ellas el contenido y la organización del trabajo, la duración y la configuración del tiempo de trabajo, el sistema de remuneraciones, las posibilidades de participación en el mejoramiento de las citadas condiciones, etc., influyen en la vida y la salud de los trabajadores.

El estrés laboral es una de las manifestaciones de tal deterioro y se puede definir como la respuesta que se pone en marcha en el individuo para hacer frente a las exigencias tanto físicas como psicosociológicas que percibe del exterior. Esta respuesta depende de una combinación de elementos diferentes como son la naturaleza de las exigencias, las características del propio individuo y el apoyo que este reciba. Una situación de estrés puede producir en el individuo:

- Cambios en el comportamiento: el individuo puede llegar a situaciones de alcoholismo, tabaquismo, alteración del sue- ño, absentismo laboral, pérdida de autoestima, conflictos interpersonales.

- Síntomas subjetivos: en situaciones de estrés pueden aparecer cefalea, tensión muscular, acidez, palpitaciones, frigidez, impotencia, sensación de falta de aire, irritabilidad, dificultad de concentración y pérdida de memoria.

- Enfermedades psicosomáticas: situaciones de estrés pueden provocar igualmente, enfermedades cardiovasculares (infarto, hipertensión); digestivas (úlcera gastroduodenal, colon irritable); cutáneas (eczema); respiratorias (reacciones asmáticas); agravamiento de enfermedades relacionadas con el sistema inmunológico.

- También son frecuentes enfermedades psíquicas como la fatiga crónica, depresión, ansiedad y neurosis.

Por otra parte, la sensación de estrés puede estar ligada no sólo a sucesos percibidos como negativos por el individuo, sino también a la falta de estímulos positivos. La variedad, la autonomía, la autorrealización y el éxito son ejemplos de situaciones positivas cuya ausencia puede ser una fuente de estrés crónico. Este estado ha sido definido como "síndrome de tedio", caracterizado como agotamiento físico, emocional y actitudinal.

En las últimas décadas muchos trabajos han descrito el estrés laboral en las profesiones "de servicios". En ellos se da cuenta de las consecuencias negativas que provocan las condiciones laborales particularmente en los ámbitos de la salud y la educación en donde las condiciones laborales y sus efectos suelen ser más críticos. Son profesiones que, en buena medida, se eligen por "vocación" subyaciendo una concepción ideal según la cual el profesional se identifica con una imagen idílica de ayuda a los demás que lo lleva a una mayor exigencia respecto de sí mismo y, por ende, a una mayor vulnerabilidad al estrés.

En los hospitales los servicios que presta el personal de enfermería presentan una serie de características que lo hace especialmente vulnerable a la aparición de estrés, siendo según Climent y Mendes Diz las tres características principales, la continuidad, la contingencia y la emotividad(2).

- La continuidad: el personal de enfermería es el que está más cerca y más continuamente con el paciente, caracterizándose por actuar ininterrumpidamente durante las 24 horas del día y durante todo el año. A esto se agrega que tradicionalmente enfermería se ha hecho cargo de todas aquellas actividades que no le pertenecen, pero que a cierta hora del día interrumpen su jornada, como las del sector admisión o las del servicio social.

- La contingencia o la necesidad de resolver problemas que surgen de forma imprevista (agravamiento o muerte de pacientes, accidentes, entre otros). 
- La alta emotividad, porque el personal de enfermería trabaja permanentemente frente al dolor y la muerte, lo cual crea un clima de gran estrés emocional.

La importancia de analizar el estrés de los profesionales de la salud surge del hecho de que el "instrumento" más importante en el cuidado de la salud de la población son los mismos profesionales.

Algunos estudios han enfocado el tema de lo que se ha dado en llamar "burnout" de los médicos y enfermeras trabajando en instituciones hospitalarias. El síndrome "burnout" es un síndrome clínico descrito en 1974 por Herbert Freudenberger $^{(3)}$, psiquiatra que trabajaba como asistente voluntario en la Clínica Libre de Nueva York para toxicómanos, al igual que otros voluntarios jóvenes e idealistas. Observó que hacia el año de empezar a trabajar, la mayoría sufría una progresiva pérdida de energía, hasta llegar al agotamiento, así como desmotivación para el trabajo, junto con varios síntomas de ansiedad y de depresión. Freudenberger describió como estas personas se vuelven menos sensibles, poco comprensivas y hasta agresivas en relación con los pacientes, con un trato distanciado y cínico, con tendencia a culpar al paciente de los propios problemas que padece. Para describir este patrón conductual homogéneo Freudenberger eligió la misma palabra, "burnout", que utilizaban también para referirse a los efectos del consumo crónico de las sustancias tóxicas de abuso. Freudenberg describe que las personas con riesgo de desarrollar burnout son "idealistas, optimistas e ingenuas", y se entrega en exceso al trabajo para conseguir "una buena opinión de sí mismo". Este tipo de motivaciones inconscientes pueden producir tan alta motivación y compromiso profesional, como también la pérdida ulterior de sus elevados y costosos ideales, el mal rendimiento laboral, la baja autoestima y el aislamiento relacional. El fracaso en la búsqueda de sentido existencial personal a través del trabajo es la causa básica de la inicial implicación laboral excesiva, como de la deserción laboral ulterior.

Posteriormente Maslach ${ }^{(4)}$ y Pines y Kafry ${ }^{(5)}$ ampliaron el enfoque, adoptando la perspectiva de la investigación social, usando cuestionarios y entrevistas. A partir de estos autores la exploración se centró en las causas y factores del escenario laboral relacionados con el burnout, tal como son percibidos por los actores que participan del escenario laboral en estudio. Sintetizando las diferentes definiciones del síndrome burnout podemos sacar cinco factores comunes:

1. Predominan los síntomas disfóricos y, sobre todo, el agotamiento emocional.

2. Destacan las alteraciones de la conducta (conducta anormal del modelo asistencial o despersonalización de la relación con el cliente).
3. Se suelen dar síntomas físicos de estrés psicofisiológico, como cansancio hasta el agotamiento, malestar general, junto con técnicas paliativas reductoras de la ansiedad residual, como son las conductas adictivas, que, a su vez, median en deterioro de la calidad de vida.

4. Se trata de un síndrome clínico/ laboral que se produce por una inadecuada adaptación al trabajo, aunque se de en individuos considerados presuntamente normales.

5. Se manifiesta por un menor rendimiento laboral, y por vivencias de baja realización personal, de insuficiencia e ineficacia laboral, desmotivación y retirada organizacional.

Por tanto se puede definir el "burnout" como una respuesta al estrés crónico con tres componentes: agotamiento emocional o físico o alguno de los dos, disminución de la productividad y despersonalización.

Para complementar el estudio psicosocial del personal de enfermería hay que tener en cuenta las posibles situaciones de acoso laboral. Ésta es una situación en la que uno o varios individuos se perciben a si mismo como objeto de persistentes acciones negativas por parte de una o varias personas, durante un prolongado periodo de tiempo. En esta situación, las personas acosadas tienen dificultad para defenderse de dichas acciones. No denominamos acoso laboral a un incidente aislado.

Existen algunas particularidades del ambiente hospitalario en relación con el estrés laboral que también merecen ser destacadas. La actividad asistencial es siempre cambiante y la dilación puede significar la pérdida de una vida. Esta situación de incontrolabilidad que hace referencia a la escasa o nula influencia que tiene el trabajador de la salud sobre el contenido y planificación de su trabajo está íntimamente vinculada con la experiencia de estrés.

Asociado a este factor aparece el de la sobrecarga o saturación de trabajo, como así la mayor exposición a riesgos y accidentes laborales debido al cansancio y a la fatiga que actúan como factores precipitantes. Por otra parte, la aparición del SIDA actúa en el mismo sentido, dado que ante cada paciente el profesional vivencia la posibilidad de contagio por el virus de la inmunodeficiencia adquirida, lo cual es altamente estresante para el personal involucrado ${ }^{(6,7)}$.

El trabajo nocturno es otro estresor significativo para el trabajador del hospital, debido a sus influencias en el ciclo biológico, como alteraciones en los ciclos circadianos y del sueño-vigilia ${ }^{(8)}$. Por otra parte, esta modalidad laboral posee también influencias sociales, por ejemplo, en la relación trabajo-familia. Este último factor es caracterizado por algunos autores como desbordamiento del ámbito laboral en el familiar(9). El personal de salud muchas veces adecua su vida al ritmo que requiere el hospital. 
El riesgo de incurrir en negligencias o impericias constituye otro de los factores estresantes del ambiente hospitalario, debido a los temidos juicios por mala práctica, actividad jurídica cada vez más creciente en muchos países ${ }^{(10)}$.

Por último, un factor prácticamente inevitable en el desempeño de enfermería es el contacto con el sufrimiento y la muerte. Esta situación suele ser fuente de problemas para la convivencia entre los integrantes del equipo de salud. El enfrentamiento diario con el deterioro humano coloca muchas veces al personal de salud en una situación en que las relaciones humanas de jerarquía y aún las científicas se ven modificadas, llegando a desvirtuarse la clara definición de derechos y obligaciones.

La profesión de enfermería exige de forma continua estar preparados técnica-científica y psicológicamente, para dar la respuesta adecuada en el momento preciso. Un estado de alerta en conocimientos científicos y técnicos, motivados por la necesidad de asumir de inmediato determinadas decisiones, informaciones complejas y diferentes, sin patrón de respuesta porque cada paciente es un mundo diferente con evolución distinta.

Por ello, el objetivo general de este trabajo es realizar una evaluación psicosocial del personal de enfermería de las unidades de nefrología de dos hospitales de la misma ciudad, siendo los objetivos específicos del estudio, describir la situación de este grupo en cuanto a clima laboral, tipo de conflictos del grupo, gestión de los conflictos, relación con el mando inmediato y bases de poder, acoso laboral y comparación entre las unidades de ambas instituciones.

\section{METODOLOGÍA}

\section{1.- Material}

Este estudio se realizó evaluando 66 encuestas contestadas por el personal de enfermería de los servicios de nefrología, de dos hospitales multifuncionales de la misma ciudad. Uno de ellos triplica la capacidad de asistencia y tiene el triple de personal.

De los 66 encuestados, 63 eran diplomados en enfermería y 3 auxiliares de enfermería. Del total de sujetos, 36 eran mujeres, 29 eran hombres y una encuesta no especificaba su condición. En cuanto a la edad, 31 sujetos tenían entre 40 y 49 años; 21 entre 18 y 29 años; 10 entre 30-39 años y finalmente 4 superan los 49 años. La edad media de los encuestados era de 37 años.

En relación con el tipo de contrato que vincula al personal encuestado con la organización, 37 disfrutaban de un contrato indefinido, 26 temporal, 2 tenían un contrato fijo discontinuo y uno era autónomo.

La mayoría tienen relación con su jefe actual desde menos de un año ( $\mathrm{n}=35)$, los demás sujetos tienen al mismo jefe desde hace 1-3 años ( $\mathrm{n}=14$ ), y de 4-10 años ( $\mathrm{n}=13$ ); sólo 3 personas mantienen una relación con su superior más de 10 años y una persona no contesta a esta cuestión.

La experiencia laboral en la organización en la que trabajan actualmente oscila entre menos de un año $(n=11)$ y más de 10 años ( $n=43$ ), sólo 7 personas llevan trabajando entre 1-3 y 4 personas entre 4-10 años. Una persona no informa de su antigüedad en la organización.

\section{2.- Método}

Este trabajo se realizó mediante un cuestionario auto aplicable experimental elaborado por la Universidad de Sevilla, en colaboración con Fremap en la línea de investigación sobre Prevención de Riesgos Laborales. El cuestionario se entregó al personal de enfermería para su cumplimentación que de forma totalmente voluntaria y anónima quiso participar. Dicho cuestionario está formado en su totalidad por 194 preguntas que se distribuyen de la siguiente forma:

- Cuestionario nº 1 de 40 ítems, que hacen referencia al trabajo en equipo o clima laboral.

- Cuestionario no 2 de 9 ítems, que evalúan las relaciones entre compañeros y jefe inmediato (conflictos del equipo de trabajo).

- Cuestionario n 3 de 28 ítems, en relación con las posibles desavenencias, incompatibilidades y diferencias en el trabajo con los demás y como se solucionan (gestión de los conflictos laborales).

- Cuestionario no 4 de 29 ítems, para conocer la opinión que los sujetos tienen de su jefe inmediato y la relación con él (bases de poder que utilizan los jefes).

- Cuestionario no 5 de 29 ítems o cuestionario NAQ sobre el tipo de acoso, comportamientos mas frecuentes sobre el tipo de acoso, etc.

- Cuestionario no 6 de 14 ítems dirigidos a conocer la situación frente al acoso laboral que padecen los sujetos en su puesto de trabajo (percepción individual).

- Cuestionario no 7 de 31 ítems o cuestionario GOLDBERG para determinar tipos de acoso no identificados por el NAQ (indicadores de disfunciones de carácter y salud mental).

Finalmente hay una relación de 8 ítems para realizar los análisis estadísticos referentes a sexo, edad, tipo de relación laboral, antigüedad, etc. 


\section{RESULTADOS}

Clima organizacional: El clima de la organización en la que trabajan se caracteriza predominantemente por ser un clima de apoyo (media $=5.25)$ y reglas (media $=5.33)$. Es decir el personal conoce la forma en la que tiene que actuar ante las situaciones más frecuentes. Las puntuaciones más bajas se relacionan con los climas de innovación (media $=2.64$ ) y metas $($ media $=2.58)$.

Tipo de conflicto: Los sujetos manifiestan que en sus equipos de trabajo el conflicto de tarea (media $=2.91$ ) es algo más elevado que el conflicto afectivo (media $=2.44$ ).

Estilo de gestión del conflicto: A pesar de que los sujetos no han identificado ningún estilo de gestión del conflicto predominante (las medias no superan los 3.5 puntos), el estilo de integración (media $=3.68$ ) es el que mayor puntuación obtiene, les siguen la evitación (media 3.32), el compromiso (media $=3.28$ ), el servilismo (media $=2.87$ ) y por último el estilo de dominación (media $=2.35$ ).

Bases de poder: No existen diferencias en el empleo de bases de poder formales $($ media $=2.92)$ e informales $($ media $=$ 2.79).

Ante la definición de acoso laboral que se plantea en los cuestionarios NAQ y GOLDBERG, 51 sujetos manifiestan no haber padecido acoso en los últimos seis meses, 2 eligen la opción "de vez en cuando", uno elige "varias veces a la semana", y 12 personas no responden. Una persona es acosada desde al menos un año y 3 desde hace dos años o más. Cinco personas han sido objeto de acoso hace cinco años y 10 personas han sido testigos de acoso. La mayoría de los empleados no contestan a las cuestiones relacionadas con el acosador, es decir, si el acosador es uno o varios compañeros, uno o varios subordinados, uno o varios jefes o los clientes.

Cuestionario NAQ. En consonancia con los resultados obtenidos anteriormente en la definición de acoso laboral, los sujetos de la muestra también puntúan bajo en el NAQ. A pesar de la casi inexistente incidencia de acoso laboral, las personas identifican que el tipo de acoso en relación a asuntos de trabajo (media $=1.33$ ) es mayor que el acoso personal (media $=1.14)$.

Cuestionario GOLDBERG. Como se podía esperar, al no haber acoso laboral, los resultados del GOLDBERG muestran que los sujetos puntúan bajo en sus diferentes síntomas. Codificados con los valores "cero es igual a ausencia de síntomas" y "uno es igual a presencia de síntomas", los resultados muestran que a pesar de las bajas puntuaciones obtenidas, los sín- tomas manifestados por los empleados se pueden ordenar del siguiente modo: en primer lugar, los sujetos manifiestan tener síntomas somáticos (media $=0.21$ ), segundo, tanto ansiedad $e$ insomnio (media $=0.18)$ como disfunción social (media $=$ 0.12 ), y por último depresión grave (media $=0.03$ ).

\section{DISCUSIÓN}

Según los resultados obtenidos en las encuestas podemos afirmar que el clima de la organización donde se desarrolla el trabajo es una mezcla entre clima de apoyo y reglas. Esto quiere decir que existe un ambiente cordial donde cada uno sabe bien lo que debe hacer, influido por la juventud (edad media 37 años) ganas de aprender e ilusión por la profesión. En nuestro estudio el conflicto de tarea predomina sobre el conflicto afectivo. Esto quizás pueda estar motivado porque no estén bien definidas algunas de las tareas entre los estamentos de auxiliares y diplomados en enfermería.

El estilo de gestión para solucionar el conflicto que más aceptación tiene por parte del personal, es un estilo de integración, aunque no es totalmente definitorio de estas unidades, ya que su media apenas rebasa los 3.5 (3.68), lo que nos indica que en definitiva el estilo de gestión utilizado va a depender bastante del tipo de conflicto y de las personas o equipos inmersos en él. En consonancia, las bases de poder están distribuidas prácticamente por igual entre las formales e informales y aun existiendo jerarquías, el mando no es autoritario.

Que el acoso laboral es casi inexistente en estas unidades de trabajo, si bien en los casos que suceden se dan más sobre las funciones laborales del trabajador (falta de información para desarrollar el trabajo, críticas de mala praxis, etc.) que sobre las propias características de la persona (forma de vestir, forma de expresarse, etc.). Consecuencia de este tipo de acoso, se observa que las posibles consecuencias somáticas, de insomnio y ansiedad, de disfunción social y depresión grave; son de muy baja incidencia según se deduce del GOLDBERG.

Finalmente comentar que aunque el estudio se ha realizado en dos unidades pertenecientes a hospitales distintos de la misma ciudad, las diferencias halladas entre ellas no son significativas.

\section{CONCLUSIONES}

Las conclusiones obtenidas en nuestro estudio fueron:

1. El clima laboral es una mezcla entre clima de apoyo y reglas.

2. El tipo de conflicto que predomina en la organización es de tareas sobre el afectivo. 
3. El estilo de gestión para solucionar los conflictos es el de integración.

4. Las bases de poder están divididas entre formales e informales y el mando no es autoritario.

5. El acoso laboral es casi inexistente, siendo éste sobre la función laboral del trabajador, lo que provoca muy baja incidencia somática en el individuo.

6. Las diferencias halladas entre las dos unidades no son significativas.

\section{BIBLIOGRAFÍA}

1. Neffa J. Reconsideración de la noción de condiciones y medio ambiente de trabajo. CEIL/CONICET. Condiciones y medio ambiente de trabajo en Argentina. Humanitas, 1987.

2. Climent G, Mendes Diz A. Modelo para la definición y evaluación de la calidad del personal de enfermería. Medicina y Sociedad 1983; 6 (6).
3. Freudenberger H. Staff burnout. Journal of Social Sciences 1974; 30 (1).

4. Maslach C. Burned out. Human Behavior 1978; 15.

5. Pines A, Kafry D. Occupational tedium in the social services. Social Work 1978; 23.

6. Kornblit A, Mendes Diz A. Los trabajadores de la salud en los tiempos del SIDA. Colección CEA-CBC. UBA, 1995.

7. Rubio J, Gómez A, Durán M. Estrés ocupacional en profesionales de los servicios de salud. Revista de Psicología Social Aplicada 1991; 1(1).

8. Peiró J. Desencadenantes del Estrés laboral. Eudema Psicología Ed; 1993.

9. Piotrkowski C. Families and Work. En: Sussman, Stenmetz. Handbook of marriage and the family. NY: Plenium Press; 1987.

10. Simpson L, Grant L. Sources and Magnitude of Job Stress Among Physicians. Journal of Behavioral Medicine 1991; 14(1). 